\title{
Consulta de enfermagem no pré-natal: representações sociais de gestantes
}

Prenatal nursing consultation: social representations of pregnant women

Consulta prenatal de enfermería: representaciones sociales de mujeres embarazadas

\section{Danyella Evans Barros MeloI, Susanne Pinheiro Costa e Silva ${ }^{\mathrm{II}}$, Khesia Kelly Cardoso Matos $^{\text {III }}$, Victor Hugo Silva Martins ${ }^{\text {IV }}$}

\begin{abstract}
Resumo: Objetivo: analisar as representações sociais de gestantes acerca da consulta de enfermagem no prénatal. Método: estudo qualitativo realizado com 30 gestantes acompanhadas por enfermeiros da Saúde da Família por meio de entrevistas semiestruturadas, processadas pelo software Iramuteq, com posterior Análise de Conteúdo. Resultados: o pré-natal representou momento importante para as participantes, especialmente por possibilitar entender as descobertas acerca da formação de um novo ser, destacando-se o diálogo e orientações perpassadas pelo enfermeiro. Permite, também, elucidar a evolução da gravidez por meio de exames rotineiros e complementares, dando-lhes segurança de um desfecho saudável. Considerações finais: esta investigação demonstrou que as entrevistadas objetivaram a figura do enfermeiro como alguém que lhes passa segurança, ancorando-se na ideia de que pondo em prática aquilo que lhes é orientado, a culminância se dará com o nascimento de um bebê saudável.
\end{abstract}

Descritores: Estratégia Saúde da Família; Cuidado Pré-Natal; Assistência de Enfermagem; Enfermagem no Consultório; Gestantes

Abstract: Objective: To analyze the social representations of pregnant women about prenatal nursing consultation. Method: a qualitative study conducted with 30 pregnant women accompanied by Family Health nurses through semi-structured interviews, processed by the Iramuteq software, with subsequent Content Analysis. Results: prenatal care was an important moment for the participants, especially because it made it possible to understand the discoveries about the formation of a new being, highlighting the dialogue and orientations that the nurse passed through. It also allows to elucidate the evolution of pregnancy through routine and complementary examinations, assuring them of a healthy outcome. Final considerations: This research demonstrated that the interviewees aimed at the figure of the nurse as someone who gives them security, anchored in the idea that putting into practice what is directed to them, the culmination will occur with the birth of a healthy baby.

Descriptors: Family Health Strategy; Prenatal care; Nursing care; Nursing in the office; Pregnant women

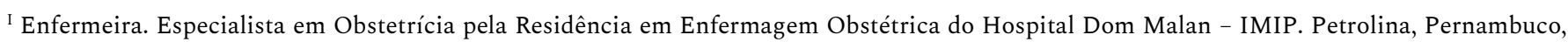
Brasil. E-mail: danyella.evans@gmail.com. ORCID: https://orcid.org/0000-0002-9021-8462

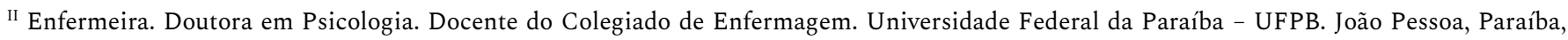
Brasil. E-mail: susanne.pc@gmail.com. ORCID: http://orcid.org/0000-0002-9864-3279

${ }^{\text {III }}$ Enfermeira. Mestre em Psicologia pela Universidade Federal do Vale do São Francisco - UNIVASF. Petrolina, Pernambuco, Brasil. E-mail: khesia.matos@gmail.com. ORCID: http://orcid.org/0000-0002-8772-8568

IV Enfermeiro. Mestrando em Educação pelo Programa de Pós-Graduação em Formação de Professores e Práticas Interdisciplinares da Universidade de Pernambuco - UPE. Petrolina, Pernambuco, Brasil. E-mail: victorugow@hotmail.com. ORCID: http://orcid.org/0000-0002-0103-9332
} 
Resumen: Objetivo: Analizar las representaciones sociales de mujeres embarazadas sobre la consulta de enfermería prenatal. Método: estudio cualitativo realizado con 30 mujeres embarazadas acompañadas por enfermeras de Family Health a través de entrevistas semiestructuradas, procesadas por el software Iramuteq, con posterior análisis de contenido. Resultados: la atención prenatal fue un momento importante para las participantes, especialmente porque permitió comprender los descubrimientos sobre la formación de un nuevo ser, destacando el diálogo y las orientaciones por las que pasó la enfermera. También permite dilucidar la evolución del embarazo a través de exámenes de rutina y complementarios, asegurándoles un resultado saludable. Consideraciones finales: Esta investigación mostró que los entrevistados objetivaron la figura de la enfermera como alguien que les da seguridad, anclados en la idea de que al poner en práctica lo que se les dirige, la culminación ocurrirá con el nacimiento de un bebé sano.

Descriptores: Estrategia de Salud Familiar; Cuidado prenatal; Cuidado de enfermería; Enfermería en la oficina; Mujeres embarazadas

\section{Introdução}

A gestação é um período de mudança biopsicossocial que ocorre na vida da mulher, pois envolve a criação e o desenvolvimento de um novo ser, sendo marcada por modificações corporais intrínsecas características dessa fase. ${ }^{1}$ Tal experiência pode ocasionar sentimentos e percepções que vão desde a alegria pela chegada de um novo membro até desconforto e medo mediante às transformações da vida. ${ }^{2-3}$

Diante da concepção é necessário que haja acompanhamento adequado, preferencialmente, desde o início da gestação, como preconiza o Ministério da Saúde. ${ }^{4}$ Tal assistência prestada a gestante durante o ciclo gravídico puerperal é garantida por meio do PréNatal. Este, por sua vez, é caracterizado como um conjunto de procedimentos cuja finalidade é promover o acompanhamento do binômio e de sua família, de forma integral e humanizada, pelos profissionais de saúde, tendo como resultado a gestação saudável e o parto sem complicações para a mãe e seu filho. ${ }^{5}$ Em vista disso, o mesmo é garantido mediante políticas públicas para monitorar fatores de risco e minimizar o efeito de possíveis complicações. ${ }^{6}$

O acompanhamento pré-natal vem sendo estimulado no âmbito dos serviços de saúde desde 1979, com a criação do Programa de Assistência Integral à Saúde da Mulher (PAISM), na tentativa de diminuir as altas taxas de morbimortalidade da gestante e concepto. O PAISM 
promoveu, então, importantes mudanças no setor, culminando em influências diretas na construção do Sistema Único de Saúde (SUS) na década de $1980 .^{6-7}$

A partir do ano 2000, foi instituído pela Portaria 569 do Ministério da Saúde o Programa de Humanização no Pré-Natal e Nascimento (PHPN), na tentativa de assegurar o acesso, melhoria da cobertura e qualidade da atenção à saúde materna. No entanto, mesmo que o Brasil venha demonstrando avanços na cobertura da assistência e do número de consultas por gestantes durante o ciclo gravídico, ainda permanece elevado o número de óbitos de mulheres e crianças por complicações da gravidez e parto. ${ }^{7}$

Perante esse cenário, em 2011, ocorreu à implementação da Rede Cegonha. Esta objetiva promover a efetivação de um novo modelo de atenção à saúde da mulher e da criança, abrangendo o Pré-Natal $(\mathrm{PN})$ e o atendimento de crianças com até 24 meses, além de estruturar a rede de atenção e reduzir a mortalidade materna e infantil, com destaque ao componente neonatal. ${ }^{8}$

Segundo recomendações do Ministério da Saúde, a assistência pré-natal deve ser realizada na Atenção Básica pela Estratégia Saúde da Família (ESF), composta por uma equipe multidisciplinar que atua em área delimitada. Tal assistência deve ocorrer por condutas acolhedoras; com o incremento de ações educativas e preventivas; na detecção precoce de patologias e de condições de risco gestacional; na formação de vínculo com o local do parto e o acesso ao serviço de saúde de qualidade, desde a atenção básica como também em níveis de maior complexidade, tendo a participação de equipe multiprofissional. ${ }^{4}$

O pré-natal adequado deve ocorrer com, no mínimo, seis consultas, iniciando no primeiro trimestre, ocorrendo duas no segundo e três no terceiro trimestre de gestação. Durante todo o acompanhamento pelo enfermeiro, são realizados exames físicos e solicitados outros de maneira complementar. Estas ações possibilitam observar o crescimento fetal e seu desenvolvimento, reduzindo o risco de intercorrências durante a gravidez. ${ }^{6-8}$ 
Consulta de enfermagem no pré-natal: representações sociais de gestantes: | 4

Além disso, no que se refere à consulta de enfermagem, é notória a sua importância na assistência à gestante. A troca de informações entre o enfermeiro, a usuária e sua família possibilita a sistematização da assistência voltada para a promoção da saúde e prevenção de doenças e agravos, promovida por meio da educação em saúde, como também ações que focam no saber e no fazer, compreendendo o cuidado do ser humano e suas particularidades. Assim, o enfermeiro contribui para boas práticas de saúde e mudança em condutas desfavoráveis ao bemestar da gestante. ${ }^{9}$

No entanto, estudos demonstram que os cuidados mínimos para uma assistência de qualidade não estão sendo realizados de maneira satisfatória. Isso pode indicar falha nos serviços de saúde, acarretando em resultados indesejáveis, como a prematuridade, contribuindo inclusive para a manutenção dos altos índices de mortalidade materna e neonatal..$^{7,10}$

Para que esse acompanhamento peculiar seja melhor compreendido, é preciso considerar as representações sociais de gestantes frente a consulta de enfermagem no pré-natal, uma vez que fatores subjetivos estão contidos na relação entre o indivíduo que recebe o cuidado e o sistema que o produz. Isso é possível por meio da Teoria das Representações Sociais (TRS). ${ }^{11}$

A utilização da TRS no Brasil incentivou pesquisas na área da saúde, especialmente na enfermagem. Ao aplicá-la em estudos desta área, contribui consideravelmente para a compreensão das representações construídas sobre o cuidado, auxiliando também no entendimento acerca de como as pessoas agem e se comportam no cotidiano, possibilitando conduzir o cuidado conforme as necessidades do outro, que é o "alvo" a quem ele se destina. ${ }^{12}$

A perspectiva da gestante sobre a assistência pré-natal pode propiciar adequações e protocolos a partir das percepções das usuárias, posto que as necessidades percebidas por elas refletem diretamente no modo como aderem a atividades preventivas e terapêuticas. Estudos que discorrem a respeito das representações sociais das gestantes sobre a assistência PN 
5 | Melo DEB, Silva SPC, Matos KKC, Martins VHS

mostraram aspectos relacionados ao acesso, participação no cuidado, adesão às consultas, bemestar, importância na saúde e discussão da oferta de recursos materiais e humanos. ${ }^{10,13-14}$

Destarte, o presente estudo apresenta relevância para a saúde pública devido dificuldades na cobertura e acompanhamento de gestantes atendidas, denotando também a importância da precocidade da assistência pré-natal para desfechos favoráveis. Para tanto, responde a seguinte questão de pesquisa: como a consulta de enfermagem no pré-natal é vista por gestantes acompanhadas em Unidades de Saúde da Família? Objetivou, então, analisar as representações sociais de gestantes acerca da consulta de enfermagem no pré-natal.

\section{Método}

Estudo descritivo e exploratório, de abordagem qualitativa, realizado em Unidades de Saúde da Família (USF) na cidade de Petrolina, Pernambuco, Brasil. Participaram desta investigação empírica mulheres gestantes cadastrada em USF que atenderam aos seguintes critérios de inclusão: estar gestante; ter idade igual ou superior a 18 anos; ter realizado pelo menos 03 consultas pré-natais, não importando o tempo de gestação, número de gestações anteriores e passado obstétrico.

O município conta com 45 Unidades de Saúde da Família, as quais abrigam 90 equipes de saúde distribuídas em zona urbana (31 equipes) e rurais (22 equipes). A seleção das USF se deu por conveniência por meio da inserção dos pesquisadores nestas enquanto campo prático de estágio curricular. A determinação do número de participantes obedeceu ao critério de saturação das informações. ${ }^{15}$ Ao final da coleta dos dados, contou-se com 30 gestantes cadastradas em 04 (quatro) USF do referido município.

A coleta de dados ocorreu entre os meses de junho a outubro de 2017, em abordagem direta na sala de espera das USF, de acordo com a demanda dos mesmos. Aceitando o convite, 
Consulta de enfermagem no pré-natal: representações sociais de gestantes: | 6

participante e pesquisadora se dirigiam a uma sala reservada. Com o objetivo de garantir a privacidade das participantes, as entrevistas ocorreram de forma individual.

Aplicou-se um roteiro de entrevista semiestruturado com cada participante, dividido em duas partes: a primeira, com questionário sociodemográfico para a caracterização das gestantes e dados relativos à saúde reprodutiva; a segunda contou com perguntas norteadoras baseadas no objetivo desta pesquisa, sendo elas: O que você acha da consulta de enfermagem no pré-natal? O que este atendimento representa para você? De que forma você acha que o acompanhamento contribui para a sua gestação? Todas as entrevistas foram gravadas em aparelho de áudio, com posterior transcrição, mediante o consentimento e após assinatura do Termo de Consentimento Livre e Esclarecido (TCLE). O tempo médio de duração de cada uma delas foi de vinte minutos.

O estudo foi aprovado por Comitê de Ética em Pesquisa e seguiu os preceitos da Resolução 466/12, do Conselho Nacional da Saúde (CAAE 66333617.6.0000.5196). As participantes estão identificadas pela letra $G$ de gestante e o número cardinais e conforme a sequência das entrevistadas, a fim de garantir o anonimato.

Os dados do questionário foram agrupados em tabelas para caracterização da amostra. O material produzido durante as entrevistas deu origem ao corpus, tratado pelo software Iramuteq. Com base nesse teste, o programa agrupa as palavras com maior ocorrência e as organiza graficamente em função da sua frequência, sendo apresentada neste estudo pela nuvem de palavras. ${ }^{16}$ Assim, por meio da análise lexicográfica, exibe contextos caracterizados pelo vocabulário compartilhado pelos participantes, identificando seu campo comum e traduzindo a mensagem, uma vez que o programa apresenta também os trechos de discursos em que as palavras agrupadas foram ditas.

O relatório gerado pelo Iramuteq classificou como relevante $77,64 \%$ do material. Pesquisadores afirmam que para garantir a estabilidade dos resultados, é aceitável a classificação 
7 | Melo DEB, Silva SPC, Matos KKC, Martins VHS

de, pelo menos, 70\% das unidades de texto. Em seguida, prosseguiu-se com a análise do corpus, que possibilitou a inferência e entendimento dos resultados gerados pelo software. ${ }^{17-18}$

\section{Resultados e discussão}

Os dados referentes ao perfil sociodemográfico e gineco-obstétrico são apresentados em tabelas. E em seguida, os termos que retratam as representações sociais sobre a consulta de enfermagem no pré-natal: O acompanhamento Pré-natal; A importância da enfermagem no Prénatal; $\mathrm{O}$ atendimento prestado por enfermeiros e Desafios enfrentados. A discussão será realizada concomitantemente à apresentação dos resultados.

A faixa etária das participantes situou-se entre 18 e 38 anos, com média de 24 anos. A maioria se autodeclarou parda e com baixa escolaridade; casadas ou em união estável (Tabela 1). Estes achados corroboram com resultados semelhantes encontrados em outros estudos, mostrando a prevalência de mulheres jovens, pardas, com companheiro, sem renda fixa e pouco escolarizadas acompanhadas no pré-natal ofertado nas Unidades básicas de saúde. ${ }^{19-21}$

Tabela 1 - Dados sociodemográficos das participantes da pesquisa. Petrolina/PE, Brasil, 2018.

\begin{tabular}{lll}
\hline VARIÁVEIS & $\mathbf{N}$ & $\mathbf{\%}$ \\
\hline Idade & 11 & 36,7 \\
$18-20$ anos & 12 & 40 \\
$21-30$ anos & 7 & 23,3 \\
$31-40$ anos & & \\
Cor & 4 & 13,3 \\
Branca & 3 & 10 \\
Amarela & 18 & 60 \\
Parda & 5 & 16,7 \\
Preta & & \\
Estado Civil & 11 & 36,66 \\
Casada & 12 & 40 \\
União estável & 7 & 23,33 \\
Solteira & & \\
Religião & 14 & 46,7 \\
Católica & 12 & 40 \\
Evangélica & 4 & 13,3 \\
Outra/Não tem & & \\
Escolaridade & & \\
\hline
\end{tabular}


Consulta de enfermagem no pré-natal: representações sociais de gestantes: $\mid 8$

$\begin{array}{lll}\text { Ensino Fundamental Incompleto } & 6 & 20 \\ \text { Ensino Fundamental Completo } & 4 & 13,3 \\ \text { Ensino Médio Incompleto } & 7 & 23,3 \\ \text { Ensino Médio Completo } & 10 & 33,3 \\ \text { Ensino Superior Incompleto } & 2 & 6,7 \\ \text { Ensino Superior Completo } & 1 & 3,3 \\ \text { Profissão } & & \\ \text { Do lar } & 16 & 53,3 \\ \text { Estudante } & 3 & 10 \\ \text { Professora } & 2 & 6,7 \\ \text { Outra } & 9 & 30\end{array}$

Fonte: Pesquisa de campo.

No que tange aos dados obstétricos (Tabela 2), encontrou-se que grande parte estava no terceiro trimestre de gestação, com média de idade gestacional (IG) de 30 semanas; 46,7\% afirmaram ter realizado 6 ou mais consultas PN, como preconizado pelo Ministério da Saúde. ${ }^{4} \mathrm{~A}$ IG na primeira consulta variou entre 6 e 20 semanas, com média de 10 semanas.

Tabela 2 - Dados gineco-obstétricos das participantes da pesquisa. Petrolina, PE, Brasil, 2018.

\begin{tabular}{|c|c|c|}
\hline VARIÁVEIS & № & $\%$ \\
\hline \multicolumn{3}{|l|}{ Número de gestações } \\
\hline Primigesta & 11 & 36,6 \\
\hline Secundigesta & 8 & 26,6 \\
\hline Multigesta & 11 & 36,7 \\
\hline \multicolumn{3}{|l|}{ Paridade } \\
\hline Nulípara & 11 & 36,6 \\
\hline Primípara & 8 & 26,6 \\
\hline Multípara & 11 & 36,6 \\
\hline \multicolumn{3}{|l|}{ 1 Consulta } \\
\hline $1^{\circ}$ trimestre & 28 & 93,3 \\
\hline $2^{\circ}$ trimestre & 2 & 6,6 \\
\hline \multicolumn{3}{|l|}{ Número de consultas } \\
\hline$<6$ consultas & 16 & 53,3 \\
\hline$\geq 6$ consultas & 14 & 46,7 \\
\hline \multicolumn{3}{|c|}{$\begin{array}{l}\text { Tipo de parto da gestação } \\
\text { anterior }\end{array}$} \\
\hline Vaginal & 12 & 40 \\
\hline Cesário & 4 & 13,3 \\
\hline Vaginal e Cesário & 3 & 10 \\
\hline Nenhum parto anterior & 11 & 36,7 \\
\hline
\end{tabular}

Fonte: Pesquisa de campo. 
Os dados expostos ratificam os resultados encontrados em outros estudos, revelando o predomínio do pré-natal sendo iniciado ainda no primeiro trimestre de gestação. A média de idade gestacional em torno de 10 semanas, multigestas e de mulheres com histórico de gestação anterior e parto vaginal também corroboram com os dados descritos na literatura. ${ }^{19,22-23}$

No que tange ao número de consultas pré-natais, um estudo recente observou que aqueles recém-nascidos cujas mães realizaram acompanhamento adequado, com pelo menos seis consultas, tiveram menos complicações ao nascer. Além disso, a qualidade da assistência e o diagnóstico precoce representam fatores importantes para a redução de taxas de mortalidade materna e perinatal. ${ }^{2425}$

A Figura 1 agrupa o corpus das entrevistas, apresentando os dados na nuvem de palavras gerada pelo software IRAMUTEQ. Percebe-se que os termos são posicionados de forma aleatória, de modo que os maiores apresentam maior frequência de citação, demonstrando, assim, seu destaque. Desse modo, pré-natal foi o que teve maior frequência (131 vezes), além de enfermeiro (78 vezes) e atendimento (65 vezes).

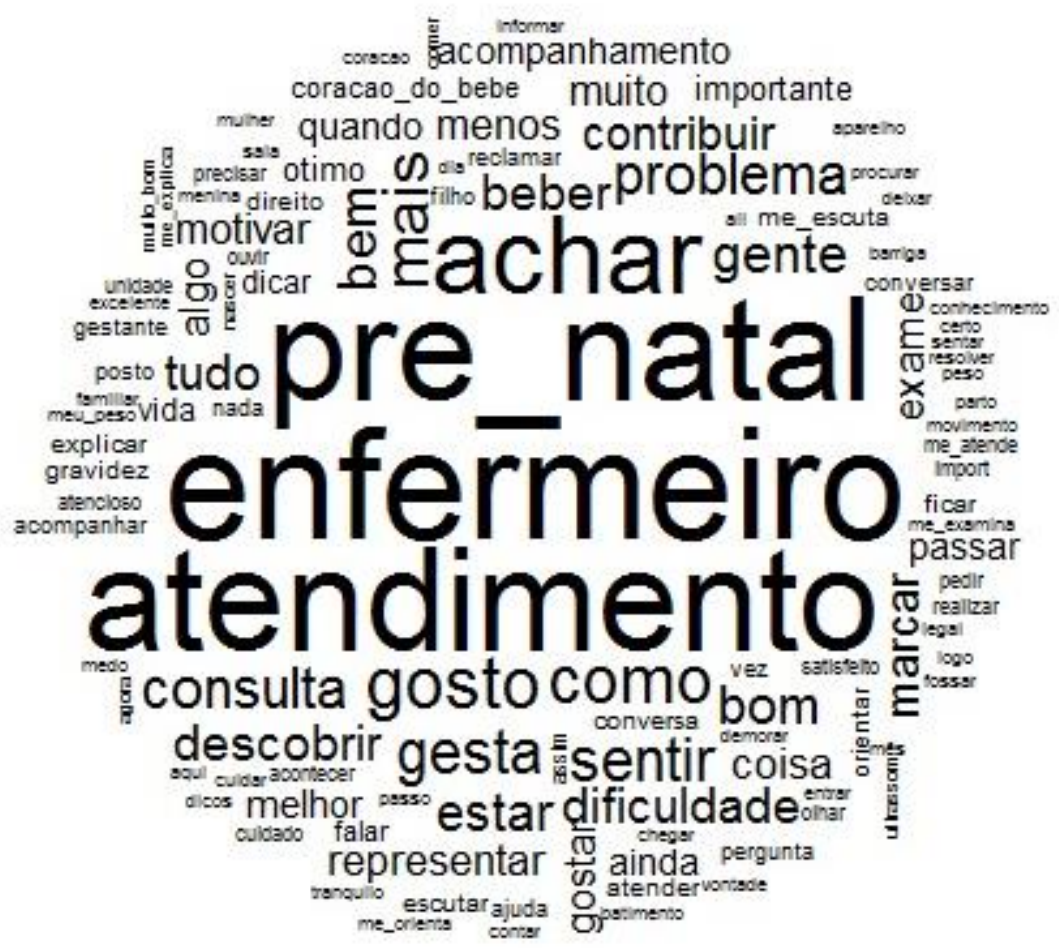

Figura 1 - Nuvem de palavras sobre a consulta de enfermagem no pré-natal para as participantes da pesquisa. Petrolina/PE, Brasil, 2018. 


\section{O acompanhamento Pré-natal}

Para fins deste estudo, após o processamento dos dados foram analisados os sentidos das palavras nos depoimentos das participantes. Desta forma, a palavra "pré-natal” demonstra que este acompanhamento representa um momento importante na vida da gestante, conectando-a com o filho que está em seu ventre. Os recortes a seguir exprimem tais achados:

O pré-natal representa muita coisa porque ali é o primeiro passo que estou dando da minha gravidez, e através do pré-natal eu sei o que está acontecendo com meu filho. (G23)

O pré-natal representa algo importante porque estou na reta final da minha gestação e não posso deixar de vir porque se algo acontecer com meu bebê, tem como eu saber. (G8)

O pré-natal tem como objetivo acolher a mulher no início de sua gestação, garantir o atendimento de qualidade e humanizado até seu parto e puerpério, para que o bem-estar do binômio mãe-filho seja assegurado. ${ }^{4}$ Nesse contexto, o enfermeiro deve ser capacitado para efetivar estas ações, com a finalidade de promover a assistência integral, resolutiva e qualificada à gestante, parturiente e puérpera, além de desempenhar papel importante na promoção da saúde. ${ }^{14}$

As atribuições do enfermeiro durante o pré-natal também foram reconhecidas nos depoimentos de algumas gestantes, marcando as suas representações. Referiram ser o atendimento deste profissional um diferencial para que procurem realizar as orientações perpassadas, auxiliando na regularidade das consultas.

O que me motivou a vir fazer o pré-natal foi a saúde minha e do bebê. (G12)

O que eu mais gosto no atendimento é a preocupação que ele tem com a gente. Ele fala o que pode e o que não pode. Gosto da sinceridade dele. (G11)

Percebe-se que as entrevistadas reconhecem a assistência de enfermagem como ferramenta para um melhor desfecho do parto. A forma como o cuidado é desenvolvido ao longo das consultas tem se tornado um instrumento fortalecedor do relacionamento entre a usuária e o enfermeiro. Assim, é 
fundamental que este aja como agente educador em saúde e portador de conhecimento técnicocientífico para a realização da consulta de enfermagem no pré-natal, diferenciando-se dos demais pela escuta ativa. Dessa forma, contribui para a qualidade da assistência e desfechos favoráveis.

Atendimento de qualidade e estabelecimento de vínculo entre profissional e gestante contribuem para a mudança de práticas e atitudes, tornando este momento o mais natural e menos medicalizado possível. No entanto, ainda são encontradas falhas nesta assistência, especialmente em localidades de zona rural e baixa renda, demonstrando lacunas na cobertura adequada àquelas que necessitam de cuidados e orientações por vivenciarem uma experiência única a cada gravidez. ${ }^{7}$

\section{A importância da enfermagem no Pré-natal}

No que tange o termo “enfermeiro”, ele é visto como imprescindível no atendimento prénatal já que a maioria das participantes demonstrou satisfação quanto ao profissional que as assistia, como pode ser observado adiante.

Se não tivéssemos os conhecimentos que a gente tem durante o pré-natal, acho que a gente poderia ter alguma coisa que pudesse trazer algum mal à saúde da bebê. (G29)

A enfermeira contribui porque ela ensina como amamentar, os cuidados que tem que ter, principalmente para as gestantes de primeira viagem, porquê tira dúvidas. (G20)

Eu sugiro que as consultas sejam de 15 em 15 dias e não de mês em mês. São muito importantes! (G30)

O enfermeiro tem papel imprescindível no $\mathrm{PN}$, operando como simplificador e pluralizador de conhecimento. A consulta de enfermagem funciona como ocasião para que se cultive o vínculo com a gestante. Além disso, o enfermeiro deve instruir a gestante e sua família sobre a importância do pré-natal, amamentação, vacinação e periodicidade das consultas; solicitar exames complementares de acordo com protocolos estabelecidos; fazer testes rápidos; realizar abordagem sindrômica de infecções sexualmente transmissíveis (IST); prescrever 
medicamentos padronizados para o programa pré-natal; promover atividades educativas; realizar exames clínico das mamas e o Papanicolau, entre outras atividades. ${ }^{24-25}$

Algumas das participantes, ainda, sugeriram que o intervalo entre as consultas fosse diminuído para que pudessem tê-las em número maior, mesmo no pré-natal de baixo risco. O fato de manifestar interesse por mais consultas e em pouco espaço de tempo pode demonstrar a indispensabilidade de um cuidado voltado para as suas necessidades. Visto isso, a consulta de enfermagem pode contribuir de forma positiva nessas situações, quando há carência de informações prévias sobre a gestação e cuidados que devem ser tomados, possibilitando segurança.

\section{$O$ atendimento prestado por enfermeiros}

Em relação ao termo "atendimento”, seu sentido esteve especialmente voltado à relação interpessoal da gestante com o profissional durante a assistência pré-natal, sendo este um momento em que os diálogos são estabelecidos e permitem que, mais do que um profissional, o enfermeiro seja visto como alguém da confiança delas.

O que eu mais gosto no atendimento da enfermeira é o diálogo, porque ela conversa bem, gosta de escutar e se preocupa. (G23)

Eu gosto porque ela não se coloca como profissional; é como uma amiga. Gosto mais com ela do que com o médico porque me sinto mais à vontade. (G14)

Percebe-se que a interação com o enfermeiro pelo diálogo, acolhimento e preocupação deste com a saúde da gestante faz com que a usuária se sinta mais segura. Condutas como acolhimento e escuta qualificada são as atividades que as gestantes expectam da equipe que atende no pré-natal. Tais meios, conhecidos como tecnologias leves, aperfeiçoam o cuidado e possibilitam que o ser seja visto integralmente em suas necessidades. ${ }^{10}$

$\mathrm{Na}$ percepção das gestantes, o atendimento de qualidade está mais ligado à atenção voltada no momento da consulta, ao diálogo e orientações que o profissional disponibiliza do que aos procedimentos técnicos. Assim, elas se preocupam com as informações recebidas 
durante a assistência e tendem a comparecer mais às consultas e outras atividades oferecidas pela equipe de saúde. ${ }^{13}$

Diante disso, é notório que as representações sociais de gestantes acerca da consulta de enfermagem pré-natal indicam que, para elas, este atendimento é visto como essencial para desfechos favoráveis no parto e pós-parto. É por meio deste acompanhamento que são esclarecidas as dúvidas e prestadas às ações para prevenir agravos possíveis, especialmente para aquelas que não possuem experiência prática no assunto. Sendo assim, é importante que tais profissionais reconheçam a dimensão do cuidado que realizam, buscando cada vez mais trabalhar de forma humanizada e acolhedora.

\section{Desafios enfrentados}

Apesar dos posicionamentos atribuídos ao contexto pré-natal/enfermeiro/gestante, na nuvem de palavra aparece um termo relativamente mencionado nas entrevistas e que apresenta pertinência para os resultados encontrados. O elemento é “problema”, cuja frequência foi de 44 repetições. No que concerne a este termo, seu sentido atribuiu-se às dificuldades e conflitos enfrentados no pré-natal.

Um dos problemas que vejo é a demora! Porque ele demora a atender uma gestante. Então, a gente tem que esperar muito se não chegar cedo. (G21)

Uma coisa que poderia ser melhorada é a estrutura das salas e os aparelhos, que são antigos. Podiam ser mais modernos. (G17)

Eu vejo como problema que os médicos e enfermeiros poderiam ser mais atenciosos para que o atendimento seja ainda melhor, porque há pouca atenção e também o posto [Unidade Básica de Saúde], que é muito lotado. (G3)

Percebe-se nos depoimentos das mesmas que um dos desafios enfrentados para o atendimento na unidade de saúde é a grande demanda e estrutura do serviço. Isso acarreta tempo de espera maior do que o necessário e, na maioria dos casos, os profissionais necessitam ser ágeis para atender a tempo, o que, muitas vezes, dificulta o estabelecimento de vínculo e a 
possibilidade do diálogo. Alguns achados foram encontrados em outros estudos, em que parte relatam o aumento no tempo de espera e falta de acolhimento, por parte do enfermeiro da equipe de saúde, à escuta qualificada. 5,10,23

Outro estudo explora a perspectiva de enfermeiros a respeito dos desafios enfrentados no pré-natal, realidade cotidiana da enfermagem, que recebe muitas solicitações das gestantes para pouco tempo de atendimento. Merece destaque o fato deste acompanhamento requerer consulta individualizada, com cuidado e atenção à gestante. Para dar conta, os profissionais acabam realizando consultas mais rápidas, perdendo a qualidade do atendimento..$^{5,10-23}$

Observam-se inúmeros desafios e esforços para garantir um atendimento pré-natal de qualidade. Por isso, as Representações Sociais tornam-se elementos importantes na avaliação deste de acordo com cada realidade social. O que, para algumas, é um agradável momento de esclarecimentos, para outras acaba sendo apenas uma consulta que não abarca a integralidade pela falta de vínculo com o profissional que a assiste. Vale destacar que o acolhimento ofertado pelos enfermeiros é fundamental para que a relação entre este e a comunidade seja estabelecida.

\section{Considerações finais}

Conhecer as representações sociais de gestantes atendidas nas Unidades de Saúde da Família permitiu compreender o quanto valorizam o atendimento pré-natal realizado por enfermeiros, especialmente pela possibilidade de chegarem ao fim da gestação sentindo-se preparadas. Os dados encontrados reafirmam que este é uma importante ferramenta, sendo indispensável pelas orientações perpassadas, escuta ativa - que permite mais que o simples ouvir: trocar informações - e acompanhamento da evolução da gravidez, demonstrando que se sentem cuidadas.

Sabe-se que o pré-natal não se baseia apenas em consultas e solicitação de exames. Realizar estratégias que envolvam o acolhimento e o reconhecimento das necessidades de saúde contribui para o estabelecimento de vínculo entre gestante, equipe de saúde e família. 
No presente estudo, as participantes objetivaram a figura do enfermeiro como alguém que lhes passa segurança, ancorando-se na ideia de que pondo em prática aquilo que lhes é orientado, a culminância se dará com o nascimento de um bebê saudável. Mesmo assim, coloca em pauta a necessidade de melhorias na gestão do atendimento devido a questões intrínsecas à equipe de saúde e gestores, como a disponibilidade de tempo e estrutura de qualidade.

Assim, os achados deste estudo poderão contribuir para melhor compreensão da importância atribuída ao enfermeiro, além da imagem deste estar atrelada à educação em saúde. Por estes e outros motivos, os enfermeiros devem buscar cada dia mais conhecimentos teórico-práticos para melhor assistirem a seu público, culminando em valorização da assistência e transformação de suas práticas.

O presente estudo contribui para a discussão entre as equipes de saúde, na medida em que explora a complexidade do cuidado ofertado à gestante na Atenção Básica, demonstrando a necessidade de reavaliação de atitudes frente a sua clientela. No entanto, apresenta como fatores limitantes o fato de ter sido realizado apenas sob a perspectiva das usuárias, além da investigação ter ocorrido em um único município brasileiro, dificultando generalizações. Sugere-se a realização de novos estudos a respeito da temática sob o olhar de diferentes contextos e sujeitos sociais.

\section{Referências}

1. Silva LS, Pessoa FB, Pessoa DTC, Cunha VCM, Cunha CRM, Fernandes CKC. Análise das mudanças fisiológicas durante a gestação: desvendando mitos. Rev Eletrônica FMB [Internet]. 2015 [acesso em 2016 dez 01];8(2):1-16. Disponível em: http://revista.fmb.edu.br/index.php/fmb/article/view/11/8

2. Velho MTAC, Riesgo I, Zanardo CP, Freitas AP, Fonseca R. A reincidência da gestação na adolescência: estudo retrospectivo e prospectivo em região do sul do Brasil. Saúde e Pesqui. 2014;7(2):261-73.

3. Tomaschewski-Barlem JG, Bordignon SS, da Costa CFS, Costa CDO, Barlem ELD. Promovendo a autoestima na gestação: foco no acolhimento. Enferm Foco (Brasília) [Internet]. 2016 [acesso em $2016 \mathrm{dez}$ 01];7(2):83-6. Disponível em: http://revista.cofen.gov.br/index.php/enfermagem/article/view/801/326

4. Ministério da Saúde (BR). Atenção ao pré-natal de baixo risco: série A: normas e manuais técnicos Cadernos de Atenção Básica, $n^{\circ}$ 32. Brasília (DF): Secretaria de Atenção à Saúde, Departamento de 
Consulta de enfermagem no pré-natal: representações sociais de gestantes: | 16

Atenção Básica; 2012 [acesso em 2017 fev 20]. Disponível em: http://bvsms.saude.gov.br/bvs/publicacoes/cadernos_atencao_basica_32_prenatal.pdf

5. Assunção CS, Rizzo ER, Santos ME, Basílio MD, Messias CM, Carvalho JB. O enfermeiro no pré-natal: expectativas de gestantes. Rev Pesq Cuid Fundam [Internet]. 2019 [acesso em 2019 nov 26];11(3):576-81. Disponível em: https://pdfs.semanticscholar.org/ff7b/05e3fdc0f6d048ddedcffc4cc5fc6efd6ff9.pdf

6. Domingues RMSM, Dias MAB, Leal MC, Gama SGN, Theme-Filha MM, Torres JA, et al. Adequação da assistência pré-natal segundo as características maternas no Brasil. Rev Panam Salud Pública. 2015; 37:140-7.

7. Martinelli KG, Santos Neto ET, Gama SGN, Oliveira AE. Adequação do processo da assistência prénatal segundo os critérios do Programa de Humanização do Pré-natal e Nascimento e Rede Cegonha. Rev Bras Ginecol Obstet. 2014;36:56-64.

8. Brasil. Ministério da Saúde. Secretaria de Atenção à Saúde. Departamento de Ações Programáticas Estratégicas. Portaria no 1459, de 24 de junho de 2011. Institui no âmbito do SUS a Rede Cegonha [Internet]. Brasília (DF): Ministério da saúde; 2011 [acesso em 2017 fev 20]. Disponível em: http://www.ibfan.org.br/legislacao/pdf/doc-693.pdf

9. Rodrigues SCA, Silva KCO, Rodrigues APA, Ribeiro TCO. Ações educativas desenvolvidas pelo enfermeiro durante o pré-natal em uma Unidade Básica de Saúde. Rev Interdiscip [Internet]. 2015 [acesso em 2019 nov 26];8(2): 68-74.

Disponível

em:

https://revistainterdisciplinar.uninovafapi.edu.br/index.php/revinter/article/view/731

10. Ramos ASMB, Almeida HFR, Souza IBJ, Araújo MCM, Pereira PSL, Fontenele R M. A assistência pré-natal prestada pelo enfermeiro sob a ótica das gestantes. Rev Interdiscip [Internet]. 2018 [acesso em 2019 nov 26];11(2):87-96. Disponível em: https://dialnet.unirioja.es/servlet/articulo?codigo=6763719

11. Camargo BV, Schlösser A, Giacomozzi AI. Aspectos epistemológicos do paradigma das representações sociais. In: Medeiros ED, Araújo LF, Coutunho MPL, Araújo LS, organizadores. Representações sociais e práticas psicossociais [Internet]. Curitiba: CRV; 2018 [acesso em 2019 nov 26]. p. 47-60. Disponível em: http://www.europhd.net/sites/default/files/camargo_b._v._scholsser_a._giacomazzi_a.i._2018._aspectos_episte mologicos_do_paradigma_das_representacoes_sociais.pdf

12. Ferreira MA. Teoria das representações sociais e contribuições para as pesquisas do cuidado em saúde e de enfermagem. Esc Anna Nery Rev Enferm [Internet]. 2016 [acesso em 2017 de fev 14];20(2):2149. Disponível em: http://www.scielo.br/pdf/ean/v20n2/1414-8145-ean-20-02-0214.pdf

13. Ortiga EPF, Carvalho MDB, Pelloso SM. Percepção da assistência pré-natal de usuária do serviço público de saúde. Rev Enferm UFSM [Internet]. 2015 [acesso em 2018 jan10];5(4):618-27. Disponível em: https://periodicos.ufsm.br/index.php/reufsm/article/view/13230

14. Martins QPM, Ferreira GSM, Aragão AEA, Gomes FMA, Araújo LM, Ferreira FIS. Conhecimentos de gestantes no pré-natal: evidências para o cuidado de enfermagem. Sanare (Sobral) [Internet]. 2015 
17 | Melo DEB, Silva SPC, Matos KKC, Martins VHS

[acesso em 2017 dez 22];14(02):65-71. Disponível em: https://sanare.emnuvens.com.br/sanare/article/viewFile/827/498

15. Minayo MCS. Análise qualitativa: teoria, passos e fidedignidade. Ciênc Saúde Colet [Internet]. 2012 [acesso em 2019 dez 12];17(3):621-6. Disponível em: https://www.scielosp.org/scielo.php?pid=S1413$81232012000300007 \&$ script $=$ sci_arttext\&tlng=en

16. Kami MTM, Larocca LM, Chaves MMN, Lowen IMV, Souza VMP, Goto DYN. Trabalho no consultório na rua: uso do software IRAMUTEQ no apoio à pesquisa qualitativa. Esc Anna Nery Rev Enferm [Internet]. 2016 [acesso em 2018 jan 10];20(3):e20160069. Disponível em: http://www.scielo.br/scielo.php?script=sci_arttext\&pid=S1414-81452016000300213

17. Kronberger N, Wagner W. Palavras-chave em contexto: análise estatística de textos. In: Bauer MW, Gaskell G. Pesquisa qualitativa com texto, imagem e som: um manual prático. Petrópolis (RJ): Vozes; 2002. p. 416-41.

18. Coutinho MPL, Serafim RCNS, Araújo LS. A aplicabilidade de desenho-estória com tema no campo da pesquisa. In: Coutinho MPL, Saraiva ERA. Métodos de pesquisa em psicologia social: perspectivas qualitativas e quantitativas. João Pessoa (PB): Ed. Universitária; 2011. p. 205-50.

19. Corrêa MD, Tsunechiro MA, Lima MOP, Bonadio IS. Avaliação da assistência pré-natal em unidade com estratégia saúde da família. Rev Esc Enferm USP [Internet]. 2014 [acesso em 2017 dez 22];48(N Esp):24-32. Disponível em: http://www.journals.usp.br/reeusp/article/view/103025/101300

20. Nascimento VF, Silva JES, Borges AP, Terças ACP, Lemes AG, Silva RGM. Percepção de puérperas sobre as primeiras consultas de pré-natal no interior de Mato Grosso. Rev Enferm UFPI [Internet]. 2016 [acesso em 2017 dez 20];5(1):46-51. Disponível em: http://www.ojs.ufpi.br/index.php/reufpi/article/view/5050/pdf

21. Rodrigues IR, Rodrigues DP, Ferreira MA, Pereira MLD, Barbosa EMG. Elementos constituintes da consulta de enfermagem no pré-natal na ótica de gestantes. Rev RENE [Internet]. 2016 [acesso em 2018 fev 19];17(6):774-81. Disponível em: http://periodicos.ufc.br/rene/article/view/6492

22. Melo LPT, Pereira AMM, Rodrigues DP, Dantas SLC, Ferreira ALA, Fontenele FMC, et al . Representações de puérperas sobre o cuidado recebido no trabalho de parto e parto. Av Enferm [Internet]. 2018 [acesso em 2018 fev 22];36(1):22-30. Disponível em: http://dx.doi.org/10.15446/av.enferm.v36n1.63993

23. Krause KMO, Alves GG, Gedrat DC, Martins MIM. Percepção das gestantes sobre o pré-natal em um centro de atendimento do interior do sul do Brasil. Aletheia [Internet]. 2017 [acesso em $2018 \mathrm{fev}$ 15];50(1):21-37. Disponível em: http://www.periodicos.ulbra.br/index.php/aletheia/article/view/4159/2986

24. Silva MIC. Avaliação da assistência pré-natal e os desfechos materno e neonatal de adolescentes que pariram numa unidade de referência no Recife, PE, 2018 [dissertação]. Recife: Fundação Oswaldo Cruz, 
Consulta de enfermagem no pré-natal: representações sociais de gestantes: | 18

Centro de Pesquisas Aggeu Magalhães; 2018 [acesso em 2019 nov 26]. Mestrado Acadêmico em Saúde Pública. Disponível em: https://www.arca.fiocruz.br/handle/icict/33035

25. Araújo RB, Anjos MRR, Souza CLO, Rodrigues TS. Cuidados de Enfermagem no pré-natal de baixo risco na Estratégia de Saúde da Família: uma análise de periódicos nacionais. Rev Uningá [Internet]. 2019 [acesso em 2019 nov 26];56(S2):160-73. Disponível em: http://revista.uninga.br/index.php/uninga/article/view/2419

\section{Autor correspondente}

Danyella Evans Barros Melo

E-mail: danyella.evans@gmail.com

Endereço: Rua Santa Inês, n 10, Vila Eulália, Petrolina, Pernambuco, Brasil.

CEP: $56.331-100$.

\section{Contribuições de Autoria}

\section{Danyella Evans Barros Melo}

Concepção e planejamento do projeto de pesquisa; obtenção, análise e interpretação dos dados; redação e revisão crítica.

\section{Susanne Pinheiro Costa e Silva}

Concepção e planejamento do projeto de pesquisa; análise e interpretação dos dados; redação e revisão crítica.

\section{Khesia Kelly Cardoso Matos}

Concepção e planejamento do projeto de pesquisa; análise e interpretação dos dados; redação e revisão crítica.

\section{Victor Hugo Silva Martins}

Análise e interpretação dos dados; redação e revisão crítica; aprovação final da versão a ser publicada.

\section{Como citar este artigo}

Melo DEB, Costa e Silva SP, Matos KKC, Martins VHS. Consulta de enfermagem no pré-natal: representações sociais de gestantes. Rev. Enferm. UFSM. 2020 [Acesso em: Ano Mês Dia]; vol.10 e18: 1-18. DOI: https://doi.org/10.5902/2179769237235 